\title{
Credibility clusters, preferences, and helpfulness beliefs for specific forms of psychotherapy
}

\author{
Rolf Sandell, David Clinton, Josefin Frövenholt and Maria Bragesjö
}

\section{Linköping University Post Print}

N.B.: When citing this work, cite the original article.

This the authors' version of the original publication:

Rolf Sandell, David Clinton, Josefin Frövenholt and Maria Bragesjö, Credibility clusters, preferences, and helpfulness beliefs for specific forms of psychotherapy, 2011, Psychology and Psychotherapy: Theory, Research and Practice, (84), 4, 425-441.

http://dx.doi.org/10.1111/j.2044-8341.2010.02010.x

Copyright: British Psychological Society http://www.bps.org.uk/

Postprint available at: Linköping University Electronic Press

http://urn.kb.se/resolve?urn=urn:nbn:se:liu:diva-74164 
Credibility clusters, preferences, and helpfulness beliefs for specific forms of psychotherapy

\author{
Rolf Sandell \\ Linköping University \\ David Clinton \\ Karolinska Institutet \\ Josefin Frövenholt \\ Maria Bragesjö \\ Linköping University
}

2010

Correspondence should be addressed to Rolf Sandell, Fredrikshovsgatan 3A, SE-115 23 Stockholm, Sweden, e-mail: rolf.sandell@liu.se 
Background: There is some evidence that the perceived credibility of specific forms of psychotherapy, beliefs about helpful and not-so-helpful interventions, and differential treatment preferences may influence both therapeutic alliance and outcome, at least in the short-term. The present study explored whether distinct clusters exist, based on credibility ratings, and how these may be related to treatment preferences and beliefs about the helpfulness of specific interventions.

Method: Written descriptions of psychodynamic (PDT), cognitive (CT) and cognitivebehavioural (CBT) psychotherapy were rated by a random community sample with the "normal" level of psychiatric treatment $(\underline{\mathrm{n}}=121)$, psychiatric outpatients with limited previous experience of psychiatric care $(\underline{\mathrm{n}}=118)$, and a group of psychiatric "veterans" with long experience of psychiatric care $(\underline{n}=48)$. Participants completed a questionnaire focusing on beliefs about the helpfulness of specific therapy components, rated the credibility of each form of psychotherapy, and ranked the alternatives in terms of preferences..

Results: Using cluster analysis six distinct groups of participants were delineated. Some approached psychotherapy in an undifferentiated manner, tending to either embrace all or reject all of the methods examined. Others had differentiated ideas about the credibility of specific therapeutic approaches. These clusters were strongly associated with differential treatment preferences. They were also associated with helpfulness beliefs, previous experiences with psychotherapy, gender, and age.

Conclusions: For clinicians it may be important to investigate the differential appeal of specific components of psychotherapy as well as entire therapy packages in individual cases prior to commencing therapy. For researchers it may be important to consider whether outcome studies would become more informative by taking patients' beliefs and preferences into account. 
Credibility, preferences, and helpfulness beliefs for specific kinds of psychotherapy

Given the placebo potential implied, the credibility of a treatment to an individual patient, his or her expectations and hopes with that treatment, his or her preferences for it, and his or her beliefs about what might be beneficial in a treatment should be an important group of common factors to explore in relation to therapeutic alliance and outcome. One problem about this group of concepts is the definitional confusion among them (Grantham \& Gordon, 1986). Thus, for example, terms like acceptability, predilection, credibility, perceived helpfulness, outcome expectations, and preferences have all been used in research that, superficially at least, seems to deal more or less with the same phenomenon, whether a person or a patient believes a certain treatment is the most reasonable or suitable option for him or her and would choose accordingly if given a choice. And it is probable - and has been demonstrated - that operationalizations of these concepts are highly correlated (Wanigaratne \& Barker, 1995). As a consequence of this confusion a review of research in this area will have to disregard, within reasonable limits, which of the terms is being used (Arnkoff, Glass \& Shapiro, 2002).

One line of research has involved asking persons (lay, students, or patients) to state their preferences among specific brands of psychotherapy or more general approaches or styles. Such surveys in various samples have consistently showed psychological treatments to be preferred to pharmacological ones (Banken \& Wilson, 1992; Chilvers et al., 2001; DwightJohnson, Sherbourne, Liao \& Wells, 2000; Riedel-Heller, Matschinger \& Angermeyer, 2005; van Schaik et al., 2004). In comparisons among psychological treatments, cognitive (CT) or cognitive-behavioural therapy (CBT) have regularly been preferred by a majority (Bragesjö, Clinton \& Sandell, 2004; Hardy et al., 1995; Tarrier, Liversidge \& Gregg, 2006; Wanigaratne \& Barker, 1995); besides, one study favoured interpersonal therapy (Banken \& Wilson, 1992). Studies of people's attitudes toward more general approaches or orientations have showed mixed results, in one study favouring cognitive and behavioural ones (Pistrang \& 
Barker, 1992), in another a "feeling orientation" (Atkinson, Worthington, Dana \& Good, 1991).

Among determining factors, several researchers have pointed to sex or gender (Atkinson et al, 1991; Banken \& Wilson, 1992; van Schaik et al, 2004), education, and socioeconomic status (Dwight-Johnson et al., 2000; Riedel-Heller et al., 2005, van Schaik et al., 2004). Age, marital status, ethnicity, and urban living have also been reported in single studies (DwightJohnson et al., 2000; Riedel-Heller et al., 2005; van Schaik et al., 2004). In general, preference for psychological treatments has been found higher among females, the highly educated, people with higher socio-economic standard, and those living in cities. Beliefs relating to problem definition and illness causes are also significant predictors, according to Riedel-Heller et al. (2005). Finally, the person's knowledge about, or familiarity with, illness and treatment options, his or her own treatment experiences and satisfaction with these is a cluster of factors that seems quite influential (Banken \& Wilson, 1992; Frövenholt, Bragesjö, Clinton \& Sandell, 2007; Tarrier et al., 2006; van Schaik et al., 2004).

Another approach in the study of preferences has focused on their relation to treatment outcome or its correlates. One design, the Match/No-Match design (M/NM; Swift \& Callahan, 2009) has been to assess preferences among treatment alternatives and then compare matched cases with non-matched or even mismatched ones in randomized designs. A variant is to compare cases in randomized trials (RCTs) that were matched or non-matched by chance. Besides comparative studies of general therapeutic styles or approaches (Duckro \& George, 1971; Goates-Jones \& Hill, 2008), which failed to show any relation between outcome and preferences, Adamson, Sellman and Dore (2005) and van Dyck and Spinhoven (1997) reported no differences between matched and non-matched cases in specific treatment comparisons. On the other hand, positive or partially positive results have been reported by Addis and Jacobson (1996), Devine and Ferneld (1973), Elkin et al (1989), Gaston, Marmar, Gallagher and Thompson, 1989; Hardy et al. (1995), and Kocsis et al. (2009) on treatment outcome and by Elkin et al. (1999) and Iacoviello et al. (2000) on treatment alliance.

In a different design, the partially randomized preference trial (PRPT), patients have been offered to choose between two options: to be randomized to alternative treatments or have their own choice between them. Only Chilvers et al. (2001) could report outcome differences in partial favour of the preference arm; Bakker, Spinhoven, van Balkom, Vleugel and van Dyck (2000), Bedi et al. (2000), King et al. (2005) and Van et al. (2009) did not. In their recent meta-analysis Swift and Callahan (2009) found that this design significantly 
underestimated the preference effect on treatment outcome in comparison with the other designs $(r=.07 ; d=0.14$ vs. $r=.20 ; d=0.41$ in $\mathrm{M} / \mathrm{NM}$ or $\mathrm{RCT})$. When drop-out from treatment was concerned, clients who received their preferred treatment were significantly less likely to drop out compared with clients who did not $(\mathrm{OR}=0.58$; Swift \& Callahan, 2009). Although the studies that reported drop-out rates were too few to seriously compare different designs, OR was higher in the PRPTs than in the other designs ( 0.91 vs. 0.73 in $\mathrm{M} / \mathrm{NM}$; 0.35 in RCTs)

In consequence of this mixture of findings, Arnkoff, Glass \& Shapiro (2002) in their review concluded that the field is insufficiently explored, empirically, and that the research that has anyway been done often has been of low quality. It remains to systematically analyze the conflicting studies in search of moderating variables. Two candidate variables are the measurement (rating, ranking, forced choice, revealed) of the concept of preferences (or whichever corresponding concept), and the distinctiveness of the treatment options. Also, more work remains to be done on the determinants of preferences. Thus, the case is certainly not closed and further research is needed, not least to empirically distinguish between concepts that are used inconsistently and interchangeably. This study is concerned with those of credibility and preferences, also introducing another one, helpfulness beliefs.

Credibility of a particular form of psychotherapy refers to a person's judgments of aspects of the viability and rationale of the method for alleviating psychological distress. Brief scales are typically used to assess it (Borkovec \& Nau, 1972; Elkin et al., 1989; Gibbons et al., 2003). Borkovec and Nau's conception is paradigmatic, focusing on whether the treatment principles make sense, whether one would be inclined to choose it, if need be, or recommend it, and on one's outcome expectations in relation to various clinical states.

However, every form of psychotherapy is a package of various specific and nonspecific components, of which some may appear - and indeed be-more helpful to the individual patient and some less so. Whereas the concept of credibility refers to a patient's evaluation of an entire package of psychotherapy, helpfulness beliefs, as we choose to call them in this study_-some have called them role preferences (Arnkoff et al., 2002; Tracey \& Dundon, 1988) — refer to a patient's beliefs about the helpfulness for himself or herself of specific and non-specific factors, interventions, or ingredients to cope with and overcome psychological distress. A therapy package may be made up from any combination of such ingredients, like understanding the childhood origins of the problem; recognizing and 
analyzing automatic thoughts; ventilation of strong feelings, and so on. More examples of such helpfulness beliefs may be found in the Opinions about Psychological Problems (OPP) scales by Pistrang and Barker (1992) and the Counseling Preference Form (CPF) used by Goates-Jones and Hill (2008). We assume that the credibility of a particular form of psychotherapy is a function of the salience of various specific and non-specific factors in the package — or, really, the patient's beliefs about their salience — and his or her beliefs about the helpfulness of these factors. Recognizing the similarities of this assumption with Fishbein and Ajzen's (1983) expectancy-value model of social attitudes, we consider credibility as an attitudinal kind of variable, with a certain degree of permanence and stability.

In turn, and in line with the expectancy-value model, credibility will likely contribute to a presumptive patient's intention or decision or action to choose one method or form of therapy over others when in need. Judgments in response to ranking or paired comparison instructions before a set of different alternatives are generally referred to as preferences and will be so called in this study ${ }^{1}$; people's actual choices are called revealed preferences in economic theory. Thus, the person has to make a choice, selecting one variety of psychotherapy and deselecting others. Illustrating the confusion among concepts, two of the items in Borkovec and Nau's (1972) credibility scale are closely related to preferences: "How confident would you be in recommending this treatment to a friend...," and "If you were ... would you be willing to undergo such treatment?"

Frövenholt et al. (2007) looked more closely at the influence of experience with psychiatric treatment on the preferences for three forms of psychotherapy (CBT, CT, PDT) in three groups of subjects with different levels of such experience. These groups were a random community sample with the "normal" level of psychiatric treatment, psychiatric outpatients with limited previous experience of psychiatric care, and a group of psychiatric "veterans" with extensive experience of psychiatric care. When respondents ranked the three forms of psychotherapy, the general public tended to be somewhat indecisive although a majority of them viewed CBT as the most preferable, whereas the two patient samples tended to be less indecisive and more often ranked CT or, somewhat less frequently, CBT as their most preferred forms of treatment.

However, it is in the nature of averages and majorities to conceal individual differences. Obviously, in each of these groups, there were some preferring CBT, some CT, and some PDT, and this division ran across the sample groups in a manner that may be more 
informative of their opinions and the basis of these. Therefore, in this study, we explore how these subjects divided themselves, irrespective of sample group, in "credibility clusters," that is, in subgroups with small within-groups and large between-groups dispersions, based on their ratings of the credibility of the three forms of therapy.

We also explore how the credibility clusters may be distinguished by the helpfulness beliefs of their members. Using credibility and preference data from a previous study (Frövenholt et al., 2007) to form such clusters and new data on helpfulness beliefs, our further aim was to explore whether cluster membership was associated with the cluster members' helpfulness beliefs about various types of psychotherapy components and with various background variables, among them the participants' previous experiences of psychotherapy.

\section{Method}

\section{Participants}

Three groups of participants were included so as make us able to create a variable reflecting their experiences of having been in psychotherapy.

The general public $(n=121)$. This sample comprised respondents from a random sample of 493 individuals from the county of Östergötland, Sweden. Thus, Of the respondents, $60 \%$ were female and 40\% male. Their age distribution was: 20-30 years (29\%), 31-40 years $(17 \%), 41-50(26 \%), 51-60(28 \%), 61+(0 \%)$. Of the participants in this group $25 \%$ had some experience with any form of psychiatric treatment, and $18 \%$ with psychotherapy, specifically, either in conjunction with pharmacological treatment (7\%) or therapy only $(11 \%)$.

Newly presenting psychiatric outpatients $(n=118)$. This sample consisted of persons who were seeking treatment for psychiatric problems at one of four separate psychiatric clinics in two counties in southern Sweden, and who, during their first contact with the clinic, were deemed at all suitable for some kind of psychological treatment.

Psychological treatment was defined as any kind of systematic problem-focused interaction, including formal psychotherapy, extending over more than the intake sessions. Patients who met these criteria were selected consecutively and asked to participate in the study. Their presenting complaints reflected a wide range of, mostly neurotic, psychopathology, including anxiety disorders and phobias, depression, relationship difficulties and obsessive problems. Males were $31 \%$ and females $69 \%$, 
with an age distribution of: 20-30 years (33\%), 31-40 years (33\%), 41-50 (21\%), 51-60 $(10 \%), 61+(2 \%)$. Of the participants in this group $70 \%$ had some previous experience with psychiatric care, $31 \%$ with therapy and medication, $30 \%$ with therapy only, $9 \%$ with medication only.

Psychiatric "veterans" with long experience of psychiatric care $(n=48)$. Members were recruited from The Swedish National Association for Social and Mental Health (RSMH), an organisation with approximately 10,000 members for people with mental and social health problems. Most members have personal experience of severe psychopathology, such as schizophrenia and/or affective disorders, and have had longstanding psychiatric contacts for these conditions. In the present study prospective respondents were recruited by an advertisement in the members' newsletter, with a circulation of c. 7500 copies. In order to exclude supporting non-patient members (e.g., family), respondents were required to have been in contact with psychiatric services for help with their own problems. No other exclusion criteria were applied. Male respondents comprised $46 \%$ of the sample, while female comprised $54 \%$; $2 \%$ were less than 30 years of age, 23\% 31-40, 25\% 41-50, 27\% 51-60, and 23\% 60+ years. All participants in this group had extensive experience of various forms of psychiatric care, including psychotherapy and medication (77\%), psychotherapy only (8\%), or medication only $(15 \%)$.

Creating the psychotherapy experience scale. On the basis of information about previous experiences with psychotherapy four groups were distinguished: Group $0(n=123)$ consisted of persons who had no experience of psychiatric care at all; Group $1(n=27)$ consisted of persons with such previous experience with, predominantly, conversation with medical personnel in the context of pharmacological treatment; Group $2(n=81)$ were persons who had been in psychotherapy and pharmacological treatment. Finally, group $3(n=51)$ consisted of persons who had been in psychotherapy only. This grouping was assumed to form an ordinal scale reflecting the extent or salience of experiences with psychotherapy.

\section{Material}

A brochure produced by Stockholm Regional Health Authority was used to provide participants with descriptions of cognitive psychotherapy (CT), cognitive-behavioural therapy (CBT), and psychodynamic psychotherapy (PDT). Acknowledged experts 
within each field had reviewed and corrected the descriptions. Descriptions comprised four components: theory of psychological problems, aims and goals, method and contract, as well as the role relationship between therapist and patient. See Bragesjö, Clinton and Sandell (2004) for details of the descriptions. The sequential order of the psychotherapy descriptions was rotated so that each form of therapy was presented first, second and third equally as often.

\section{Measures}

Background data. A short background questionnaire was used to gather information on age, gender, education, type of psychological problems, and experiences with previous treatment for psychological distress.

Credibility. In an adaptation of the Borkovec and Nau (1972) scale, each psychotherapy description was rated on four dimensions: (1) Rationale, i.e. how well-founded the method appeared to be; (2) Stress, i.e. how psychologically and emotionally challenging the method seemed; (3) Recommendation, i.e. the likelihood of recommending the method to a friend or relative, should the need arise; (4) Choice, i.e. the likelihood of oneself choosing the method in question, should the need arise. The ratings utilised 5point graded scales with end-point anchors $(1=$ very little; $5=$ very much). Principal components analyses showed that Recommendation and Choice ( 3 and 4 above) each shared about $90 \%$ with the total variance of the credibility ratings. When conducting cluster analysis only these two variables were therefore used in relation to each of the three forms of psychotherapy, i.e. six variables were used to cluster the participants.

Preferences. At the end of the questionnaire participants were asked to rank the three forms of psychotherapy according to which form they would prefer (over other forms) for themselves if they were in need of psychological help. On the ranking task there was also a response alternative for "psychopharmacological treatment," as well as a "don't know" alternative. Due to a purely technical mistake, the psychopharmacological alternative was omitted in the questionnaire administered to the general public sample. Preferences were thus rendered as a rank order, with 1 as the most preferred and 4 as the least preferred alternative.

Helpfulness beliefs. The Psychotherapy Preferences and Experiences Questionnaire (PEX; Clinton \& Sandell, unpublished) is a 50-item self-report instrument to assess initial beliefs about particular types of interventions and styles of doing psychotherapy. 
Respondents are given the task of imaging that they are about to start psychotherapy and are asked to rate on four-point scales the extent to which they believe they would be helped by items reflecting both specific and non-specific ingredients in psychotherapy. Ratings are averaged in terms of five subscales with 10 items each: Externalisation (e.g. believing to be helped by "training in practical solutions to problems," "setting concrete goals," "focusing on solutions rather than causes"), Internalisation (e.g. believing to be helped by "looking for repeating life patterns," "reflecting on painful memories," "putting feelings into words"), Catharsis (e.g. believing to be helped by "loosening control," "getting strong feelings out of one's system," "expressing sorrow and anger"), Support (e.g. believing to be helped by "being encouraged," "being met with care and consideration," "feeling confirmed"), and Defensiveness (e.g. believing to be helped by "avoiding difficult situations," "keeping feelings under control," "avoiding conflicts").

The subscale structure has been supported by factor analyses. The alpha reliabilities of the subscales in the present sample were all between .85 and .86 . An earlier version of the PEX has been used successfully to examine the relationship of helpfulness beliefs and experiences to outcome in generalized anxiety disorder (Levy Berg, Sandahl \& Clinton, 2008). An English version of the PEX used in the present study is available from the authors upon request.

\section{Procedure}

General public._Potential participants received the questionnaire, descriptions of psychotherapy, and a cover letter describing the project through the post. A letter reminding participants to return their questionnaires was posted after three weeks. Because potential participants were contacted anonymously this letter was sent to all potential respondents.

Outpatients. Respondents completed questionnaires prior to their first intake interview or, at one of the clinics, by their third intake interview at the latest. No active treatment was administered to any patient at any clinic until after his or her questionnaire had been returned. The patients were informed that the treatment subsequently offered would be completely independent of whether or not, or how, the patient had answered the questionnaire.

Psychiatric veterans. Measures were posted to members who had responded to the advertisement. 
Results

\section{Credibility Clusters and Preferences}

Non-hierarchical cluster analysis applying the k-means method (Gore, 2000; SPSS 12.1) was used to analyze the credibility ratings, that is, the Recommend and Choice ratings of each of the three forms of psychotherapy. Solutions with clusters from $k=2$ to 6 were examined until a readily interpretable solution with six clusters was achieved. The profiles of each of these clusters across the six clustering variables are shown in Figure 1. The largest cluster ( $31 \%$ of participants; solid thick line) had a relatively low likelihood choosing CBT for self or recommending it to others, while being somewhat more likely to choose CT, and most likely to choose PDT or recommend it. We named this the PDT cluster. A majority (48\%) gave PDT their highest rank, 22\% CT, and 22\% chose the "don't know" alternative first on the preference task.

The second largest cluster ( $21 \%$; dashed thick line) had a relatively undifferentiated profile at a high level (i.e. overall credibility ratings above 4 on 5-point scales). We named this the Optimists cluster, because cluster members appeared to have a positive view of psychotherapy generally. The majority of the respondents in this cluster (36\%) preferred CT the most, $27 \%$ CBT, and $21 \%$ chose "don't know" on the ranking task. The PDT alternative was preferred by $16 \%$.

\section{(Insert Figure 1)}

The next cluster in terms of size (20\%) was complementary to the PDT cluster, with a high likelihood choosing CBT for self and a quite low likelihood for choosing PDT. This was named the $C B T$ cluster, with $66 \%$ of the cluster members giving CBT their first rank on the preference task. CT was preferred by $17 \%$.

Next in size were two clusters (19\% and 5\%, respectively) with high credibility ratings for CT in both. The former was named the $C T$ cluster, since there was a high likelihood of members choosing CT for their own sakes, while the latter cluster was characterised by a clear differentiation between choosing for oneself and recommending to others across all three types of therapy. In this smaller cluster, recommending CBT or PDT to a friend was much more likely than oneself choosing either, in fact even equally or more likely than choosing CT for oneself. This group was accordingly termed the CT recommendation (CTrec) cluster. On the preference ranking task 69 and $67 \%$ of these individuals, respectively, placed CT first. In 
the CT cluster $22 \%$ preferred CBT the most, in the CTrec cluster only $8 \%$ (in this small cluster meaning one person).

The final cluster (another 5\% of the participants) had a profile complementary to the Optimists, with low credibility ratings of all three forms of psychotherapy overall. We accordingly named this the Pessimists cluster. Almost half of these individuals (46\%) responded with "don't know" on the preference ranking task, whereas $31 \%$ preferred CT.

\section{Credibility Clusters, Helpfulness Beliefs, and Background Variables}

Membership in the six clusters was analyzed in relation to the PEX scales using a chi-squared interaction detection procedure (SI-CHAID v. 5; Magidson, 2005). Based on the associations among a set of predictors (as they are called in the software) and their associations with a dependent variable (clusters in this case), the total sample was stepwise split into subgroups on the predictor ("segments" in the software) with as different distributions as possible across the six clusters, generating a hierarchical tree diagram. CHAID functions in a way roughly similar to stepwise discriminant analysis; it is more powerful, however, in the sense that in each step all predictors are tested, anew and independently after each split in each branch of the tree, thus exploring local associations. Too, it has the virtue of modeling missing data in the sense of using them as another category. The five PEX subscales, each transformed into five categories in as rectangular a distribution as possible, were first included as predictors. After optimal merging of adjacent categories, most of the scales turned out to be quite predictive, in significant associations with the clusters, from Externalisation, $\chi^{2}(5 ; \underline{N}=270)=34.78$, $\underline{p}<.001$, Internalisation, $\chi^{2}(10 ; \underline{N}=270)=37.47, \underline{p}=.001$, Catharsis, $\chi^{2}(10 ; \underline{N}=270)$ $=34.26, \underline{p}=.004$, to Support, $\chi^{2}(10 ; \underline{N}=270)=30.74, \underline{p}=.014$. Given $\alpha=.05$ as eligibility and merger criteria, Externalisation was the basis for the first split (low; <4 vs. high; >3), as may be seen in Figure 2. Respondents who were low on this scale were further split on the basis of Catharsis (very low; 1 vs. not very low; >1), whereas those on high levels of Externalisation were then divided on the basis of Internalisation (very high; 5 vs. not very high; <5).

(Insert Figure 2)

These splits thus generated four segments with as different distributions across the clusters as possible within chance limits. Segment A comprised 44 persons (16\% of the sample) with low 
scores on Externalisation and very low scores on Catharsis. The CT cluster and the CBT cluster were overrepresented in this segment, in comparison with the sample as a whole. The respondents comprising Segment B (125 persons; 46\%) were also low on Externalisation but medium or high on Catharsis. Here $45 \%$ came from the PDT cluster, more than twice as many as from any other segment. Segment C (22\% of the total sample), high on Externalisation and not very high on Internalisation, had a significant overrepresentation of the CBT cluster and a slight overrepresentation of the CT cluster. Optimists comprised more than half of those in segment D (6\% of the total sample), with high scores on both Externalisation and Internalisation.

Type of psychological problems, psychotherapy experience (according to the regrouping of the respondents), respondents' age, gender, and education were then included as additional predictors. Besides the helpfulness belief scales (except Defensiveness), type of psychological problems had a significant association with the cluster distribution, $\chi^{2}$ $(5 ; N=270)=22.96, p=.004$, and so had gender, $\chi^{2}(5 ; N=270)=12.43, p=.029$, and psychotherapy experience, $\chi^{2}(5 ; N=270)=15.87, p=.049$. Thus, depression, or no particular problem at all, were particularly frequent in the CBT cluster $(80 \%)$ and among the Pessimists (100\%). Problems with anxiety, and relational problems, were both more frequent than expected in the CTrec (54\%) and Optimist (47\%) clusters. Women were the majority among the Optimists (77\%), whereas men tended to be overrepresented in the PDT and CBT clusters and among the Pessimists. When therapy experience was concerned, a significant majority of Pessimists (77\%) and the CBT cluster $(74 \%)$ had none, whereas there was a slight over-representation of persons with such experience among the Optimists (54\%) clusters. However, neither type of psychological problems or psychotherapy experience, nor gender, contributed to the division into separate segments. Instead, it turned out that age, although having no general association with the clusters, had a significant local association with the distribution of clusters in the above segment B and generated a further split, as shown in Figure $3 .^{2}$ Thus, the overrepresentation of the PDT cluster was especially pronounced in the 41-50 year group, somewhat less so in the younger groups $(-30 ; 31-40)$, and not at all in the older age groups $(51-60 ; 61+)$. Further, the frequency of Pessimists increased with age, from $2 \%$ in the younger groups, over $12 \%$ in the $41-50$ years group, to $15 \%$ in the groups over 51 years. 


\section{Helpfulness Beliefs and Preferences}

A discriminant analysis with the three psychotherapy forms (first rank on the preference task) as the grouping variable and the helpfulness beliefs as predictors yielded one significant function, $\chi^{2}(10 ; N=222)=36.25, p<.001$, with PDT and CBT at contrasting poles and CT almost linearly in-between. As might be expected, given the CHAID findings, Internalisation and Externalisation had the strongest standardized coefficients on the discriminant function, .728 and -.798, respectively. Catharsis was strongly correlated with the discriminant function (.698) but did not contribute incrementally to the between-therapies discrimination, due to its strong correlation with Internalisation (.696 within-groups). Classification on the basis of the discriminant function was correct in $44 \%$ of all cases; $61 \%$ for PDT; $55 \%$ for CBT; but only $26 \%$ for CT.

A series of logistic regression analyses, one for each form of psychotherapy, tested to what extent the PEX variables might predict whether a person would prefer it the most on the ranking task. Ranking PDT first was predicted by Internalisation, $\beta=.768$, $p=.008$, and Externalisation, $\beta=-.718, p=.005$. CBT as first rank was predicted by Externalisation, $\beta=.689, p=.002$, and - although not beyond the .5 level-by Internalisation, $\beta=-.426, p=.091$. CT, finally, was negatively predicted by Defensiveness, $\beta=-.365, p=.029$.

\section{Discussion}

The present study aimed to explore whether distinct preference subgroups for different psychotherapeutic methods would emerge across three samples of subjects. A principal finding was that distinct clusters could be identified on the basis of their credibility ratings of the three forms of psychotherapy. On the one hand, there were those who approached psychotherapy in a relatively undifferentiated manner, tending to either embrace all or reject all of the methods examined. On the other hand, there were those who appeared to constitute distinct "market segments" and have clear preferences for particular methods. Another important finding was that these clusters were predicated on the participants' beliefs of the helpfulness of specific types or classes of interventions. First and foremost, positive beliefs in treatment factors subsumed under the notions of Externalisation and Internalisation, respectively, were predictive of cluster membership. Also, previous experience with psychotherapy as well as gender 
significantly differentiated the clusters, and patients' age seemed to be of some importance in a subgroup of persons.

The relations between the helpfulness beliefs and cluster membership or choice were much as one might have expected on the basis of the theoretical ideas underlying the therapies. Thus, being assigned to the CBT cluster was most likely among persons with high scores on Externalisation and low scores on Internalisation. These individuals tended to believe to be helped by "training in practical solutions to problems," "setting concrete goals," "focusing on solutions rather than causes," and similar items but not by "exploring unconscious wishes," "becoming aware of unconscious thoughts and feelings," or "talking about embarrassing ideas." Interestingly enough, about $70 \%$ of the CBT cluster persons had no previous psychotherapy experience, and most of them were men. The PDT cluster, on the other hand, tended to be found especially among middle-aged individuals, women mostly, who were low on Externalisation and not low on Catharsis, and they did have some previous experiences with psychotherapy. They tended to expect to be helped by "loosening control", "getting strong feelings out of one's system", “expressing sorrow and anger" and the like. Choosing PDT was strongly predicted by high scores on Internalisation and low scores on Externalisation, choosing CBT by the complementary or contrasting pattern. The two came out as distinctly opposite alternatives in the discriminant analysis. When the CT cluster was concerned, it tended to be much less distinct than the other two. CT had the most appeal among respondents low on Externalisation and not high on Catharsis. The relative nonspecificity and "in-between-ness" of the CT alternative also showed in the fact that the ability to predict choice of CT was much poorer than for the other two alternatives. Somewhat paradoxically, however, CT was chosen by more respondents $(36 \%)$ in the entire sample than CBT (25\%) or PDT (21\%), especially in the two patient groups (42\%), and the two CT clusters obviously had as specific credibility profiles as the CBT and the PDT clusters. The clear distinction between CT and CBT in this study was probably a consequence of the relatively stronger emphasis on behaviour rather than cognition in the CBT text.

Two interesting subgroups of respondents delineated by the cluster analysis were the Optimists and the Pessimists. Although the majority of the Optimists tended to prefer CT, their attitudes towards psychotherapy were generally positive across the board. Optimists were particularly frequent among respondents high on Externalisation and very high on Internalisation. In contrast, the Pessimists, a relatively small cluster of respondents, appeared 
reluctant to believe that any of the three forms of psychotherapy would be helpful, and preferred to reply “don't know” when asked to rank the alternatives. These persons were particularly frequent in the older age groups with low scores on Externalisation and high scores on Catharsis. Unfortunately, since the medication alternative, by mistake, was not offered to the sample from the general public, it was not possible to examine whether this alternative was particularly appealing to the Pessimists. Although based on the small number of respondents in the two patient groups who did choose psychopharmacological treatment, there was a significant overrepresentation of these among the Pessimists (adjusted standardized residual $=4.6, p<.05)$.

When the experience factor was concerned, a different grouping than the one tested by Frövenholt et al. (2007), now pertaining specifically to psychotherapy experiences, made a significant differentiation among the clusters. It seems that it is not experience with psychiatric care in general that matters but more specifically whether the person has been in psychotherapy before or not. Those who had tended to be more optimistic about psychotherapy overall or be more specific in their choosing PDT. One may speculate that their experiences have been generally positive. People without any experience with psychotherapy on their own tended to prefer CBT. Frequent positive publicity about CBT in the mass media may be a factor influencing this group. No doubt, the texts themselves, besides the nature of the therapies, played a more important role in the credibility ratings and the preferences of participants without any experience of their own. One may surmise that CBT is more easily explained and grasped than PDT.

There were, however, a number of weaknesses with the study that should be borne in mind before considering the results more fully. Most likely, none of the samples was representative of its assumed population in any strict sense. There was a high proportion of non-response in the random sample of the general public. Even if the rate of non-response was comparable to other studies surveying the general public, those who responded were probably particularly concerned with the issues raised by the study. There was also a preponderance of women over men among newly presenting psychiatric outpatients, although this reflects the overrepresentation of women seeking psychiatric care in Sweden (Socialstyrelsen, 2001). Also, the sampling of outpatient respondents was totally at the discretion of the participating psychiatric clinics, leaving us with no information about the specific criteria for selection or about the response rates. What's more, the psychiatric veterans comprised members of a 
patient organisation who is actively engaged in questions of psychiatric care. The weak response to the advertisement in their newsletter was disappointing but may correspond to what is expected of advertising in general. However, the aim of our study was not primarily to compare the differential appeal of psychotherapy in three distinct and representative samples and make inferences to their respective populations. Instead, the groups were used to create a scale broadly representing two levels of experience with psychotherapy. Although our focus has been the comparison between three approaches to psychotherapy, the omission of a psychopharmacological alternative in the questionnaire administered to the general public was unfortunate, since it prohibited a fuller analysis of the appeal of this treatment alternative to all the groups delineated in the cluster analysis. Still another weakness was the possible consequences of the fact that our questionnaire was administered at different times in different clinics in one of the sample groups. Even the initial, brief contact with an intake interviewer may have influenced beliefs and preferences in a way to confound the effects of reading the psychotherapy presentations.

Certainly, in view of the conceptual confusion and the conflicting research results, more research is warranted. First of all, researchers (and clinicians) have to come to an agreement about the use of terms and concepts relating to people's thoughts and feelings about psychotherapy. Second, these concepts have to be operationally defined by reliable and valid instruments, and these have to be developed, tested, and improved, and their antecedents explored. Next, theories about the relations among the concepts should be advanced and tested. Expectancy-value theory (Fishbein and Ajzen, 1983) may be one such theory. The stability or the malleability of the phenomena is of course of particular interest. Exploring and testing the relations between the concepts and psychotherapy process variables like the working alliance and the real relationship (Gelso, 2009) may contribute to a theory of psychotherapy change mechanisms. Finally, the conflicting findings on the relations with therapy outcome are of course an enigma that has to be addressed. Interestingly enough, nobody seems yet to have compared randomized and self-selected therapy assignment after randomizing patients to randomization or self-selection.

Although one has to appreciate the possibility that our specific results are culture-bound, the results nevertheless suggest that it may be important for the clinician to explore the differential appeal of psychotherapy in individual cases prior to commencing therapy. As suggested by the expectancy-value model (Fishbein \& Ajzen, 1983), congruence between the 
patient's helpfulness beliefs and the therapist's treatment approach, as perceived by the patient, might be expected to increase the credibility of that approach to the patient and, we assume, to the therapeutic alliance (Iacoviello et al., 2007), possibly also treatment outcome, eventually. Thus, in conjunction with possible other indications such beliefs might be used to customize treatments (Norcross, 2002), drawing on the placebo potential implied. By enquiring about, or empirically measuring, preferences for different methods it may be possible for clinicians to identify clients who will have a predilection for a particular way of working, thereby increasing the chances of establishing a positive treatment alliance. Such actions may at least alert therapists to potential problems that may arise if the client has weak preferences for a certain way of working that the therapist tends to follow. On the other hand, some might argue that the most reasonable approach to a patient with clear beliefs and preferences may be one which the patient is not really comfortable with, because it may help her/him develop underdeveloped ways of thinking and coping. By taking beliefs or preferences into account one way or the other - and these need to be compared empiricallyit may be possible to achieve more efficacious matching of patient and treatment, thereby ultimately improving outcome. This, at least, is a possibility worthy of more systematic study, and one to which the PEX could be suited. Anyway, both clients and therapists might better profit from differentiating segments of psychotherapy patients. This could be done by using an instrument like the PEX to examine the relationship between helpfulness beliefs and the subsequent development of the treatment alliance and outcome.

What's more, it may be important to incorporate preferences into our experimental designs in order to better understand the complex interactions that influence process and outcome in psychotherapy, and better gauge the importance of treatment-specific factors relative to patient characteristics (Brewin \& Bradley, 1989; Lambert \& Wood, 2000). Working prospectively, the PEX could be used at intake to classify patients in terms of definite or indefinite preferences, allowing the former to choose their preferred forms of therapy and randomising the latter. 


\section{References}

Adamson, S.J., Sellman, J.D., \& Dore, G.M. (2005). Therapy preference and treatment outcome in clients with mild to moderate alcohol dependence. Drug and Alcohol Review, 24, 209-216.

Addis, M. E. \& Jacobson, N. S. (1996). Reasons for depression and the process and outcome of cognitive-behavioral psychotherapies. Journal of Consulting and Clinical Psychology, 64, 1417-1424.

Arnkoff, D. B., Glass, C. R., \& Shapiro, S. J. (2002). Expectations and preferences. In J. C. Norcross (Ed.), Psychotherapy relationships that work (pp. 335-356). New York: Oxford University Press.

Atkinson D. R., Worthington, R. L., Dana, D. D., \& Good, G. E. (1991). Etiology beliefs, preferences for counseling orientations, and counseling effectiveness. Journal of Counseling Psychology, 38, 258-264.

Bakker, A., Spinhoven, P., van Balkom, A. J. L. M, Vleugel, L, \& van Dyck, R. (2000). Cognitive therapy by allocation versus cognitive therapy by preference in the treatment of panic disorder. Psychotherapy and Psychosomatics, 69, 240-243.

Banken, D. M. \& Wilson, G. L. (1992). Treatment acceptability of alternative therapies for depression: A comparative analysis. Psychotherapy, 29, 610-619.

Bedi, N., Chilvers, C., Churchill, R., Dewey, M., Duggan, C., Fielding, K., et al. (2000). Assessing effectiveness of treatment of depression in primary care: Partially randomised preference trial. British Journal of Psychiatry, 177, 312-318.

Borkovec, T. D., \& Nau, S. D. (1972). Credibility of analogue therapy rationales. Journal of Behavior Therapy \& Experimental Psychiatry, 3, 257-260.

Bragesjö, M., Clinton, D., \& Sandell, R. (2004). The credibility of psychodynamic, cognitive and cognitive-behavioural psychotherapy in a randomly selected sample of the general public. Psychology and Psychotherapy, 77, 297-307.

Brewin, C. R. \& Bradley, C. (1989). Patient preferences and randomised clinical trials. British Medical Journal, 299, 313-315.

Chilvers, C., Dewey, M., Fielding, K., Gretton, V., Miller, P., Palmer, B., et al. (2001). Antidepressant drugs and generic counselling for treatment of major depression in 
primary care: Randomised trial with patient preference arms. British Medical Journal, 322,772 .

Clinton, D. \& Sandell, R. (2007). PEX - The Psychotherapy Preferences and Experiences Questionnaire: A short introduction. Unpublished manual, Stockholm: Karolinska Institutet.

Devine, D. A., \& Fernald, P. S. (1973). Outcome effects of receiving a preferred, randomly assigned or non-preferred therapy. Journal of Consulting and Clinical Psychology, 41, 104-107.

Duckro, P. N. \& George, C. E. (1979). Effects of failure to meet client preference in a counseling interview analogue. Journal of Counseling Psychology, 26, 9-14.

Dwight-Johnson, M., Sherbourne, C. D., Liao, D., \& Wells, K. B. (2000). Treatment Preferences Among Depressed Primary Care Patients. Journal of General Internal Medicine, 15, 527-534.

Elkin, I., Shea, M. T., Watkins, J. T., Imber, S. D., Sotsky, S. M., Collins, J. F., et al. (1989). National Institute of Mental Health Treatment of Depression Collaborative Research Program: General effectiveness of treatments. Archives of General Psychiatry, 46, 971982.

Elkin, I., Yamaguchi, J. L., Arnkoff, D. B, Glass, C. R., Sotsky, S. M., \& Krupnick, J. L. (1989). "Patient-treatment fit" and early engagement in therapy. Psychotherapy Research, 9, 437-451.

Fishbein, M., \& Ajzen, I. (1975). Belief, attitude, intention, and behavior: An introduction to theory and research. Reading, MA.: Addison-Wesley.

Frövenholt, J., Bragesjö, M., Clinton, D. \& Sandell, R. (2007). How do experiences of psychiatric care affect the perceived credibility of different forms of psychotherapy? Psychology and Psychotherapy: Theory, Research and Practice, 80, 205-215.

Gaston, L., Marmar, C. R., Gallagher, D., \& Thompson, L. W. (1989). Impact of confirming patient expectations of change processes in behavioral, cognitive, and brief dynamic psychotherapy. Psychotherapy, 26,_296-302.

Gelso, C. J. (2009). The time has come: The real relationship in psychotherapy research. Psychotherapy Research, 19, 278-282. 
Gibbons, M. B. C., Crits-Christoph, P., de la Cruz, C., Barber, J., Siqueland, L., \& Gladis, M. (2003). Pretreatment expectations, interpersonal functioning, and symptoms in the prediction of the therapeutic alliance across supportive-expressive psychotherapy and cognitive therapy. Psychotherapy Research, 13, 59-76.

Goates-Jones, M. \& Hill, C. E. (2008). Treatment prfeference, treatment-preference match, and psychotherapist credibility: Influence of session outcome and preference shift. Psychotherapy: Theory, Research, Practice, Training, 45, 61-74.

Gore, Jr., P. A. (2000). Cluster analysis. In H. E. A. Tinsley \& S. D. Brown (Eds.), Handbook of applied multivariate statistics and mathematical modeling (pp. 298-324). San Diego, CA: Academic Press.

Grantham, R. J. \& Gordon, M. E. (1986). The nature of preference. Journal of Counseling and Development, 64, 396-400.

Hardy, G. E., Barkham, M., Shapiro, D. A., Reynolds, S., Rees, A., \& Stiles, W. B. (1995). Credibility and outcome of cognitive-behavioural and psychodynamic-interpersonal psychotherapy. British Journal of Clinical Psychology, 34, 555-569.

Iacoviello, B. M., McCarthy, K. S., Barrett, M. S., Rynn, M., Gallop, R., \& Barber, J. P. (2007). Treatment preferences affect the therapeutic alliance: Implications for randomized controlled trials. Journal of Consulting and Clinical Psychology, 75, 194198.

Joyce, A. S., \& Piper, W. E. (1998). Expectancy, the therapeutic alliance, and treatment outcome in short-term individual psychotherapy. Journal of Psychotherapy Practice \& Research, 7, 236-248.

King, M., Nazareth, I., Lampe, F., Bower, P., Chandler, M., Morou, M., et al. (2005). Impact of participant and physician intervention preferences on randomized trials: A systematic review. Journal of the American Medical Association, 293, 1089-1099.

Kocsis, J. H., Leon, A. C., Markowitz, J. C., Manber, R., Arnow, B., Klein, D. N., et al. (2009). Patient preference as a moderator of outcome for chronic forms of major depressive disorder treated with nefazodone, Cognitive Behavioral Analysis System of Psychotherapy, or their combination. Journal of Clinical Psychiatry, 70,_354-61.

Lambert, M. \& Wood, J. (2000). Incorporating patient preferences into randomized trials. Journal of Clinical Epidemiology, 53, 163-166. 
Levy Berg, A., Sandahl, C. \& Clinton, D. (2008). The relationship of treatment preferences and experiences to outcome in generalized anxiety disorder (GAD). Psychology and Psychotherapy: Theory, Research and Practice, 81, 247-259..

Magidson, J. (2005). SI-CHAID 4.0 user's guide. Belmont, MA: Statistical Innovations Inc.

Norcross, J. C. (Ed.) (2002). Psychotherapy relationships that work. New York: Oxford University Press.

Pistrang, N., \& Barker, C. (1992). Clients' beliefs about psychological problems. Counselling Psychology Quarterly, 5, 325-335.

Riedel-Heller, S. G., Matschinger, H., \& Angemeyer, M. C. (2005). Mental disorders-who and what might help? Help-seeking and treatment preferences of the lay public. Social Psychiatry and Psychiatric Epidemiology, 40, 167-174.

Socialstyrelsen (2001). Folkhälsorapport 2001 [Report on public health]. Stockholm: National Board of Health and Social Welfare.

Swift, J. K. \& Callahan, J. L. (2009). The impact of client treatment preferences on outcome: A meta-analysis. Journal of Clinical Psychology, 65, 368-381.

Tarrier, N., Liversidge, T., \& Gregg, L. (2006). The acceptability and preference for the psychological treatment of PTSD. Behavior Research and Therapy, 44, 1643-1656.

Tracey, T. J., \& Dundon, M. (1988). Role anticipations and preferences over the course of counseling. Journal of Counseling Psychology, 35, 3-14.

Van, H., Dekker, J., Koelen, J., Kool, S., van Aalst, G., Hendriksen, M., et al. (2009). Patient preference compared with random allocation in short-term psychodynamic supportive psychotherapy with indicated addition of pharmacotherapy for depression. Psychotherapy Research, 19, 205-212.

van Dyck, R., \& Spinhoven, P. (1997). Does preference for type of treatment matter? Behavior Modification, 21, 172-186.

van Schaik, D. J .F., Klijn, A. F. J., van Hout, H. P. J., van Marwijk, H. W. J., Beekman, A. T. F., de Haan, M., \& van Dyck, R. (2004). Patients' preferences in the treatment of depressive disorder in primary care. General Hospital Psychiatry, 26, 184-189.

Wanigaratne, S., \& Barker, C. (1995). Clients' preferences for styles of therapy. British Journal of Clinical Psychology, 34, 215-222. 
Credibility clusters, preferences, and helpfulness beliefs 


\section{Footnotes}

${ }^{1}$ This differs from how Arnkoff, Glass \& Shapiro (2002) use the term in their review. They define preferences a desired or valued behaviour or attribute of the therapist and differentiate between three kinds, role preferences, preferences for type of therapy, and preferences for demographic features of the therapist. Further, some of the investigations they offer as examples rather belong to what is here called credibility studies (e.g., Addis \& Jacobson, 1996; Devine \& Fernald, 1973; Hardy et al., 1995).

${ }^{2}$ Although a predictor (like age) may not be significantly associated with cluster assignment in the total sample, there may be a significant local association in a subgroup providing the basis for further division of this subgroup. 
Figure Captions

Figure 1. Mean profiles of each of the six clusters across the choice and recommendation ratings by therapy form.

Figure 2. Figure 2. SI-CHAID-generated tree diagram relating the six clusters to the PEX variables (Externalisation, Internalisation, Catharsis, Support, Defense. The PEX variables were all recoded to five-category distributions with as rectangular distributions as possible. \# refers to cluster number. Segment $[\mathrm{X}]$ refers to groups of combinations of predictors.

Figure 3. SI-CHAID-generated tree diagram relating the six clusters to the PEX variables (Externalisation, Internalisation, Catharsis, Support, Defense), experience group, gender, and age. The PEX variables were all recoded to five-category distributions with as rectangular distributions as possible. \# refers to cluster number. Segment $[\mathrm{X}]$ refers to groups of combinations of predictors. 


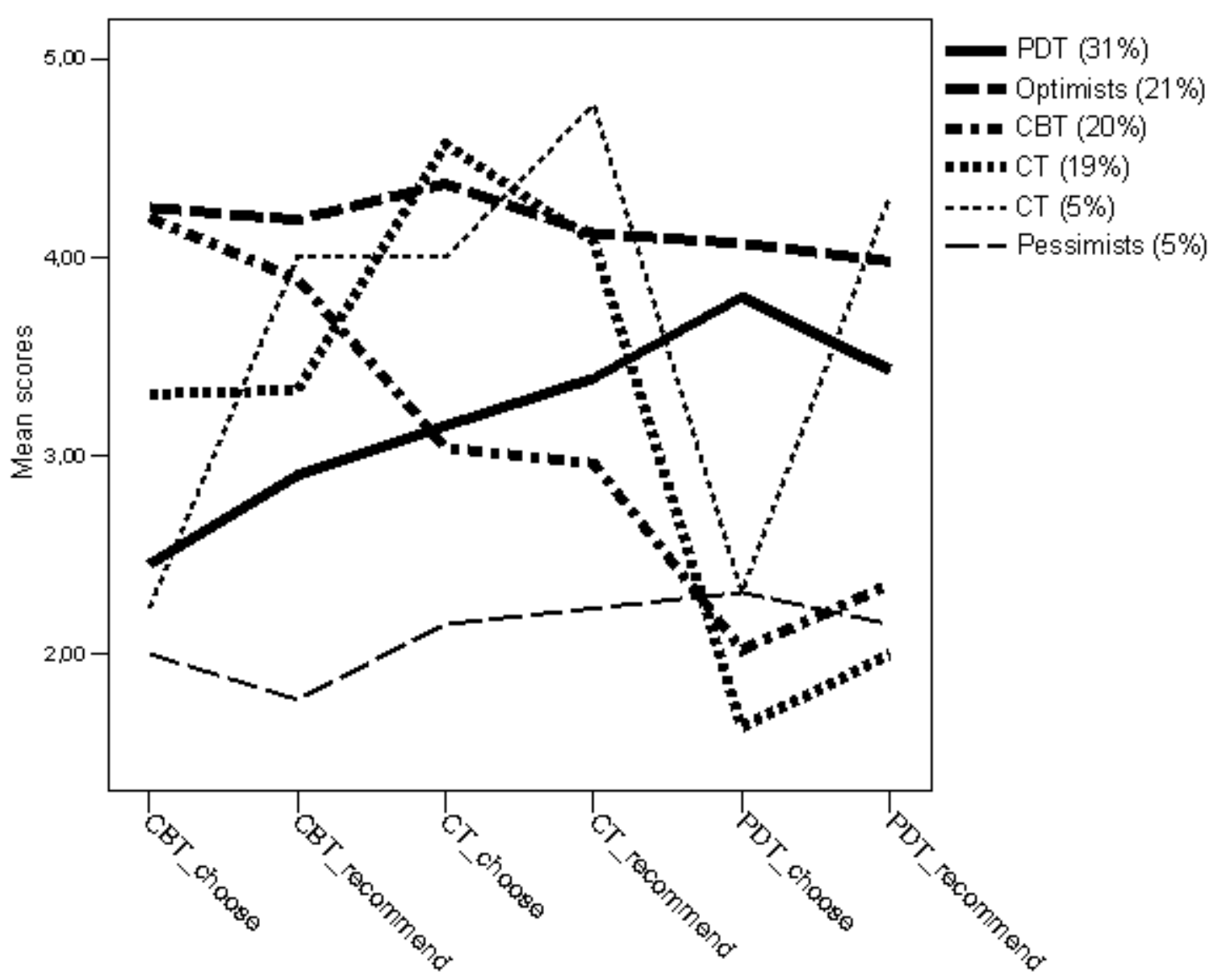




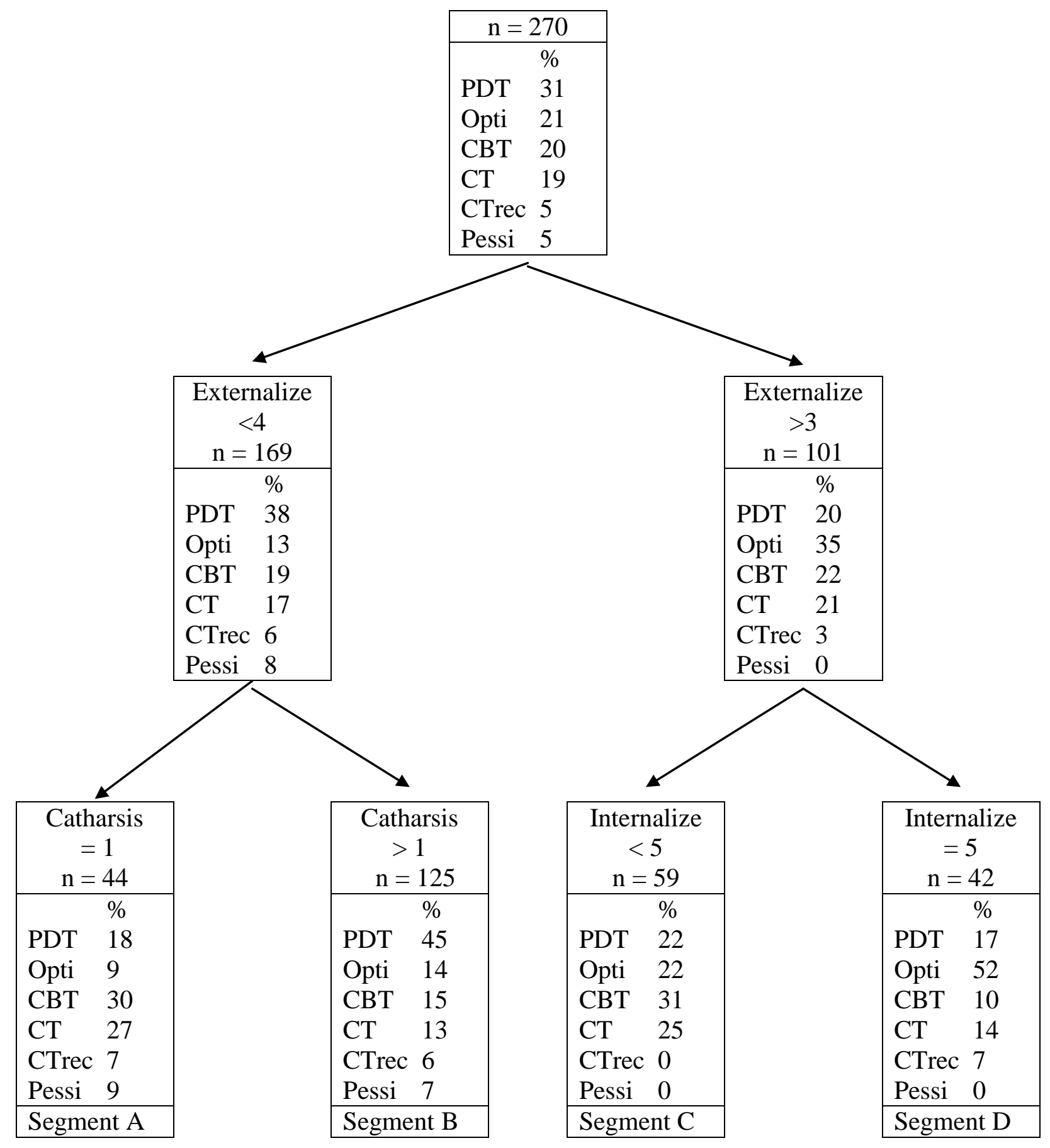




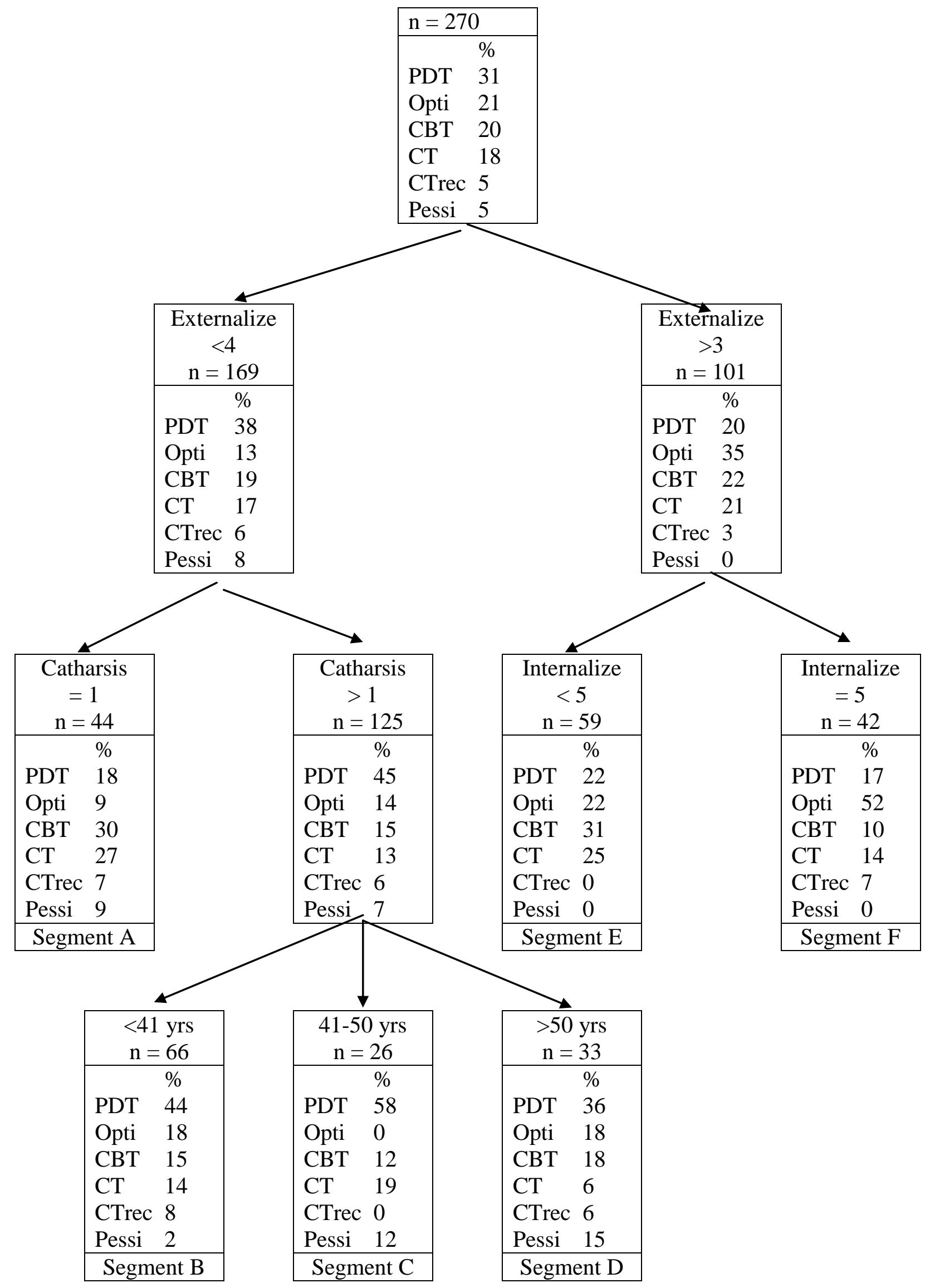


Credibility clusters, preferences, and helpfulness beliefs 